\title{
SIMULATION OF AN UNDERWATER ACOUSTIC COMMUNICATION CHANNEL CHARACTERIZED BY WIND-GENERATED SURFACE WAVES AND BUBBLES
}

\author{
HS Dol TNO, Acoustics \& Sonar department, The Hague, The Netherlands, \\ MA Ainslie henry.dol@tno.nl, michael.ainslie@tno.nl \\ MEGD Colin TNO, Acoustics \& Sonar department, The Hague, The Netherlands, \\ J Janmaat_mathieu.colin@tno.nl, jeroen.janmaat@tno.nl
}

\section{INTRODUCTION}

Sea surface scattering by wind-generated waves and bubbles is regarded to be the main nonplatform-related cause of the time variability of shallow acoustic communication channels. Simulations for predicting the quality of acoustic communication links in such channels thus require adequate modelling of these dynamic sea-surface effects. It is known that, for frequencies in the range $1-4 \mathrm{kHz}$, the effect of bubbles on sea surface reflection loss is mainly due to refraction, which can be modelled with a modified sound-speed profile accounting for the bubble void fraction in the surface layer (Hall-Novarini model). The upward refraction induced by the bubble cloud then effectively acts as a catalyst for increasing the rough-surface scattering.

In the present work, it is shown that, for frequencies in the range $4-8 \mathrm{kHz}$, bubble extinction also provides a significant contribution to the surface loss, including both the effects of bubble scattering and absorption. As this is the frequency band adopted in the European Defence Agency (EDA) project RACUN (Robust Acoustic Communication in Underwater Networks [1]), in which the reported research has been conducted, both bubble refraction and extinction effects should be modelled for acoustic channel simulations in RACUN. These model-based channel simulations will be performed by applying a Gaussian-beam ray-tracer (BELLHOP), together with a toolbox for generation of realistic rough sea surfaces based on both fully-developed ocean and short-fetch North Sea wave-height spectra and angular spreading functions (WAFO).

\section{SEA SURFACE MODELLING}

\subsection{Introduction}

The objective of the present work is to improve channel modelling for underwater acoustic communication by incorporation of the effects of time-varying ambient conditions, especially windgenerated sea surface wave effects. These effects are probably the main cause of time-varying multipath and Doppler spread when both the transmitter and receiver are static. The sea surface dynamics can roughly be divided into two basic mechanisms:

1. Periodic vertical motion of the sea surface;

2. Near-surface bubbles created by (breaking) waves.

See Figure 1 for a schematic illustration of these effects.

In order to keep things practical, we will at this point assume a separation of time scales for the sea surface dynamics and the underwater acoustic propagation. That is, we will treat the problem as "piece-wise frozen". For each "frozen" realization of the sea surface and the bubble distribution, acoustic computations are then performed without accounting for the instantaneous velocity of the sea surface and the bubbles. The main Doppler effects come in as a consequence of the variation of the path lengths between consecutive realizations [2]. This is called the range rate: $d L_{k}(t) / d t$, with 


\section{Proceedings of the $11^{\text {th }}$ European Conference on Underwater Acoustics}

$L_{k}(t)$ the distance from transmitter to receiver along a connecting sound path $k$. In this way, the sea surface modelling 'reduces' to accounting for surface roughness at different spatial scales and for the presence of air bubbles in the propagation modelling. Both mechanisms will be addressed in this paper and suitable modelling approaches are proposed.

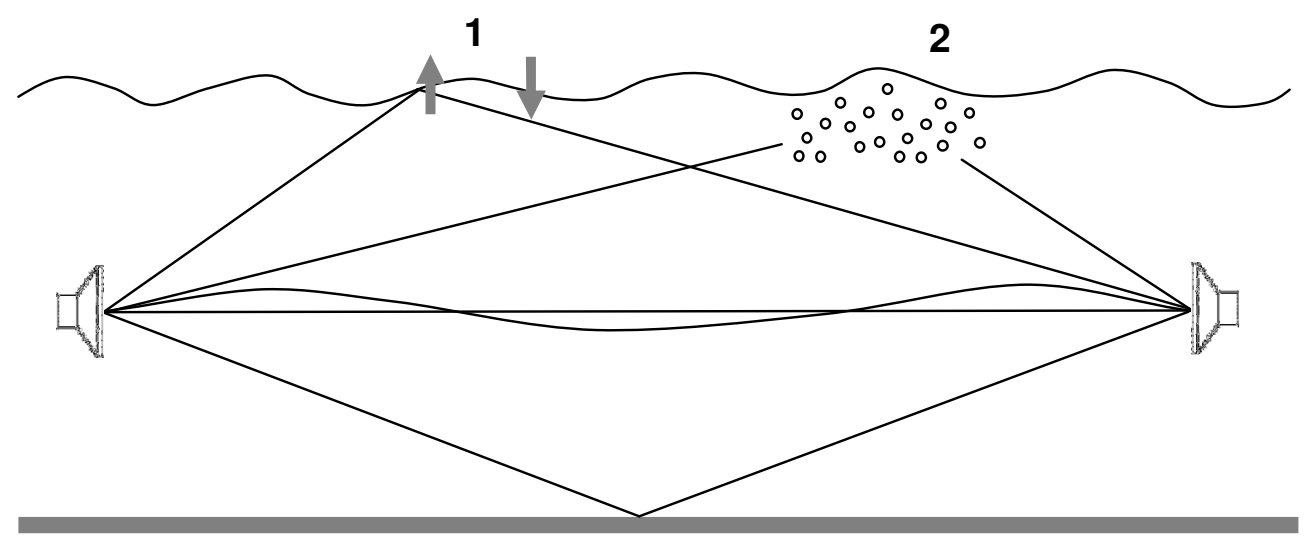

Figure 1. Illustration of the two main sea surface scattering mechanisms.

Wind-generated bubbles become significant above Beaufort wind force 3 , corresponding to wind speeds (at $10 \mathrm{~m}$ height) above $5 \mathrm{~m} / \mathrm{s}$. For developed seas (long fetch lengths), this corresponds roughly to significant wave heights of $1 \mathrm{~m}$ and higher and sea states of 2-3 and higher [3]. This is supported by measurements of Thorpe (1982) [4], performed for various wind conditions in a lake (Loch Ness, Scotland). These results show that at a wind speed of $6 \mathrm{~m} / \mathrm{s}$, the bubble layer is a few metres thick.

The following sections will consider the options for modelling of rough surfaces typical for the sea surface conditions as described above, and for modelling of the effect of the related bubble layer. As both mechanisms are active above wind speeds of $5 \mathrm{~m} / \mathrm{s}$, a practical approach will be an integrated approach. The combined sea-surface modelling will be integrated with the BELLHOP ray-tracer [5]. The reasons for selecting this propagation model are the ability to apply rangedependent surface heights and its low computational complexity (at least, for single frequencies).

\subsection{Modelling of surface waves}

As described by Siderius and Porter (2008) [6], it is possible to specify an almost arbitrary seasurface height distribution in BELLHOP. The main limitations are that breaking waves are not supported, i.e. only a single height value for each position, and that the height distribution should be one-dimensional (1D). Although the primary source of the near-surface bubbles is the airentrainment due to breaking waves, the sea surface realizations will be derived from wave spectra that are based on observed/measured significant wave heights, and details of overtopping waves are lost in these statistical descriptions. The second limitation requires some consideration. On the spatial scales of the acoustic wavelengths of interest, i.e. $15-40 \mathrm{~cm}$ for the RACUN band of $4-8 \mathrm{kHz}$, the sea-surface height distribution is intrinsically two-dimensional (2D), giving rise to out-of-plane scattering effects. The occurrence of long Doppler-spread reverberation tails, as observed in experiments for the $10-18 \mathrm{kHz}$ band [7], suggest that out-of-plane scattering effects may be important for communication channels. However, taking this into account would require threedimensional (3D) acoustic computations, which is outside the scope of the RACUN project. Instead, a vertical cross-section is made at the line from transmitter to receiver. 


\section{Proceedings of the $11^{\text {th }}$ European Conference on Underwater Acoustics}

To obtain realistic wave height distributions for rough-surface scattering, we use established wave height spectra from literature, such as Pierson-Moskowitz (PM) [8] for a fully developed sea (Atlantic Ocean), and JONSWAP [9, 10] for short fetch lengths (North Sea). Convolution with an angular spreading function, to account for variability of the wind direction, results in directional wave spectra, providing the 2D structure of the wave. An example of a realistic sea surface is shown in Figure 2. This surface realization has been created with the WAFO toolbox for Matlab $[11,12]$ using the JONSWAP wave height spectrum and a suitable (frequency-dependent) angular spreading function. Time-evolving sea surfaces can also be generated with this toolbox.

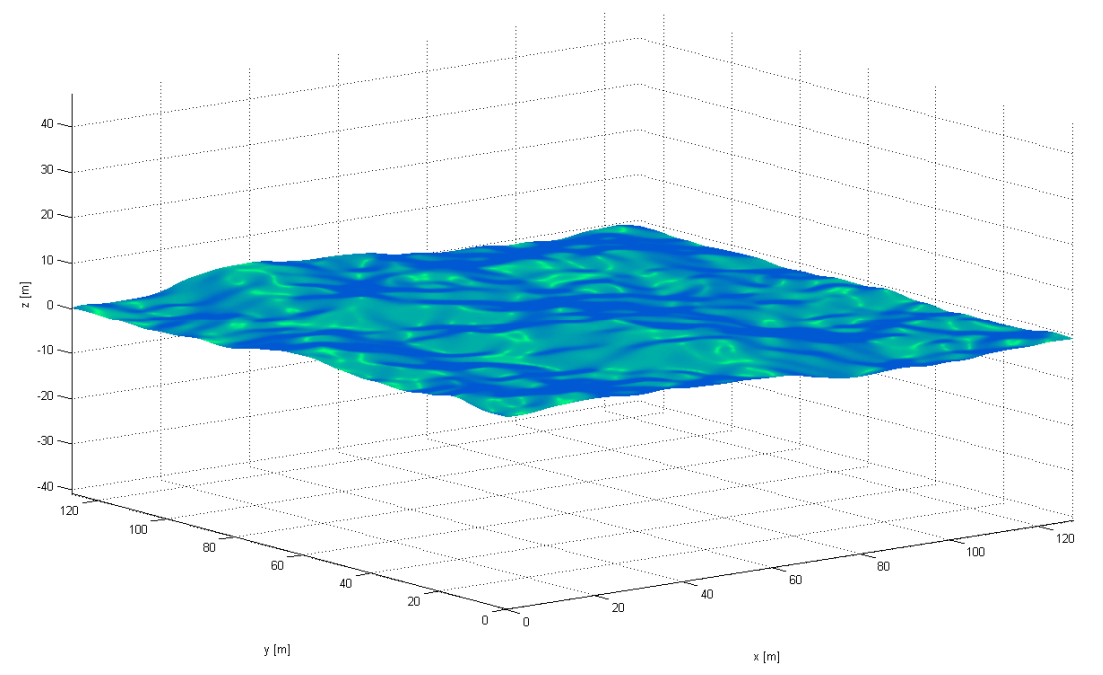

Figure 2. Example of a 2D sea surface realization using the (free) WAFO toolbox for Matlab [11]. This specific realization uses the JONSWAP spectrum, with significant wave height $H_{1 / 3}=7 \mathrm{~m}$ and modal wave period $T_{0}=11 \mathrm{~s}$, and "cos- $2 \mathrm{~s}$ " angular spreading.

\subsection{Modelling of bubbles}

We now turn our attention to modelling of the second mechanism, i.e. the effect on sound propagation of wind-generated bubbles in the surface layer. This effect can be further subdivided in three physical mechanisms:

A. Refraction, due to the sound-speed profile (SSP) being changed by the bubble void fraction;

B. Forward and backward scattering;

C. Absorption, together with the scattering denoted as extinction (of coherent propagation).

Small bubbles have a longer lifetime than large bubbles. The bubble population spectral density decreases rapidly for bubble sizes above $1 \mathrm{~mm}$, corresponding to acoustic resonance frequencies below $3 \mathrm{kHz}$. Small and large bubbles are both relevant to RACUN, but through different mechanisms. Small bubbles provide a large contribution to the void fraction, and are thus mainly responsible for refraction. Larger bubbles, especially those that resonate in the $4-8 \mathrm{kHz}$ band (up to $1 \mathrm{~mm}$ radius), are important for backscattering. The role of these large bubbles in forward scattering has yet to be investigated. Research at TNO $[13,14]$ has concentrated on the Hall-Novarini (HN) bubble population model $[15,16,17]$. This model treats the near-surface bubbles as a diffuse bubble cloud. The HN bubble spectrum includes only bubbles of radius $<1 \mathrm{~mm}$, which complies with the RACUN frequency band. The $\mathrm{HN}$ model has been used to calculate the signal strength of backscattered paths. For underwater acoustic communication, the coherence of forward scattered paths is of significance. In the present research, modelling of the forward scattering is still missing. The contribution of forward scattering will become more important when wind speed and frequency increase, and it will be interesting to see whether we can do without it here $(4-8 \mathrm{kHz}$, up to $10 \mathrm{~m} / \mathrm{s})$. 


\section{Proceedings of the $11^{\text {th }}$ European Conference on Underwater Acoustics}

For integration with the propagation modelling (i.e. BELLHOP), a practical way is to take into account the presence of a bubble cloud by means of a horizontally-averaged bubble field, taking care to avoid step changes in sound speed (smoothly varying air void fraction). A more advanced approach would be to model also the presence of bubble plumes [18], but this would largely complicate the space and time dependence while it remains to be seen whether such local and discrete phenomena would have a significant structural effect. Therefore, as a compromise between accuracy and efficiency, bubbles will be modelled here as horizontally diffuse clouds and not as discrete plumes.

An important remaining question is whether bubble extinction, i.e. the combined effect of absorption and scattering by bubbles, is a significant effect at communication frequencies, compared to refraction. Ainslie (2005) [13] showed that extinction due to the bubble cloud has a significantly smaller impact than refraction on coherent reflection loss of a plane wave in a semi-infinite water layer in the range $1-4 \mathrm{kHz}$. Coherent reflection loss is the reflection loss due to bubble extinction and rough-surface scattering, when compared to the specular reflection at a smooth bubble-free surface. The effect of bubble refraction is enhanced sea-surface interaction as the refraction is usually directed upward because (at low frequency, i.e. below resonance) the presence of a high air void fraction leads to a low sound speed.

In the present study, the results of Ref. [13] are extended up to $10 \mathrm{kHz}$, see Figure 3. Each panel in this figure shows the coherent reflection loss $-20 \log _{10}|R|$ vs. frequency and grazing angle for a wind speed at $10 \mathrm{~m}$ height of $10 \mathrm{~m} / \mathrm{s}$ ( $\mathrm{rms}$ roughness $\sigma=0.6 \mathrm{~m}$ ). $|R|$ is defined as the ratio of the mean reflected pressure amplitude to the incident amplitude. The average is usually over an ensemble of different realizations of a randomly rough surface, but it can also be thought of as an average in time over a randomly varying rough surface. The physics behind these computations is explained in Refs. [19, 13, 3].
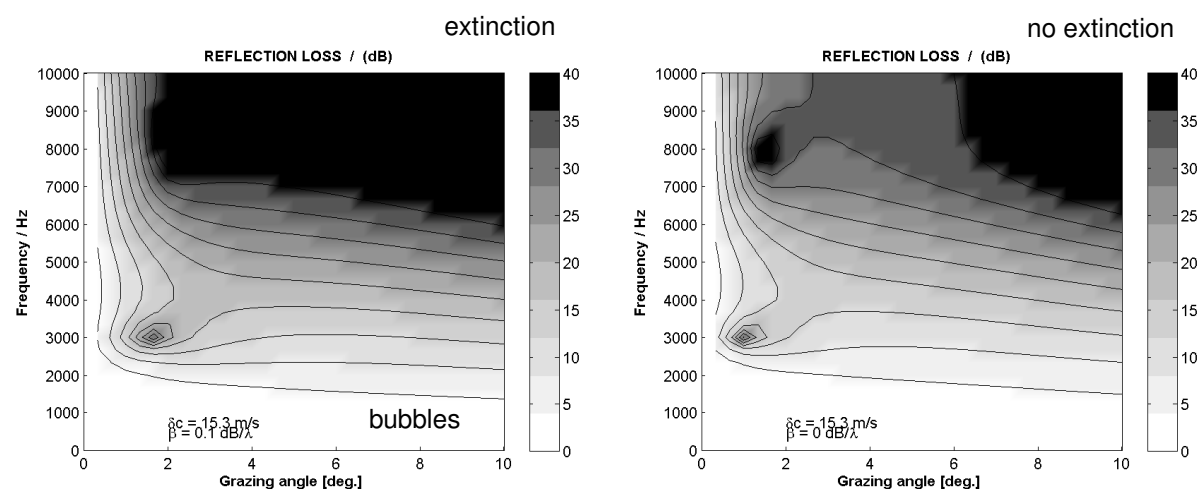

refraction
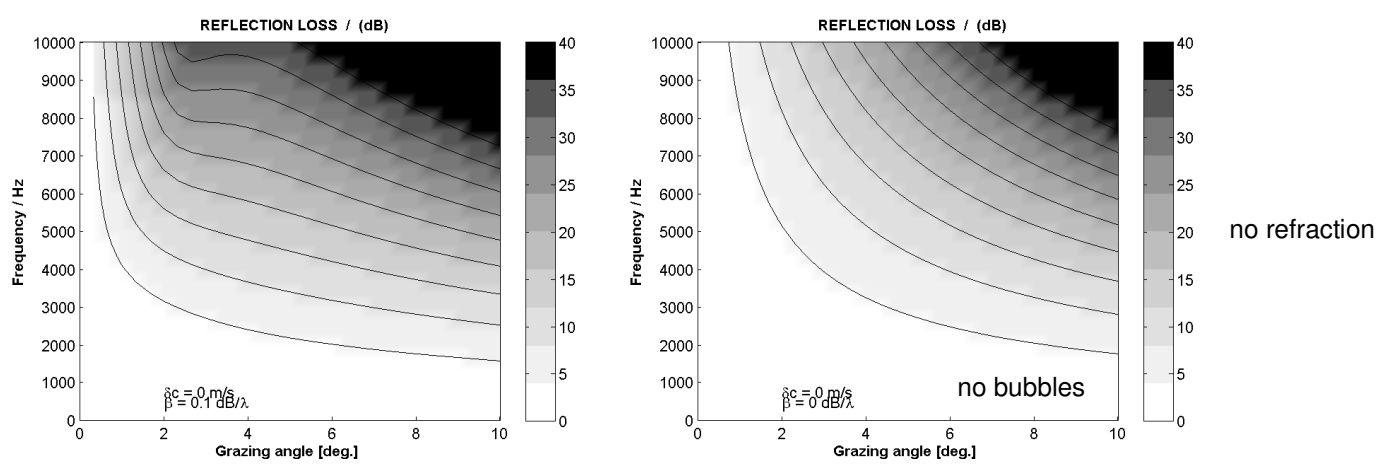

Figure 3. Coherent reflection loss $-20 \log _{10}|R|$ (in $\mathrm{dB}$ ) vs. angle and frequency for a wind speed of $10 \mathrm{~m} / \mathrm{s}$. Left-hand panels include extinction, right-hand panels exclude extinction. Upper panels include refraction, lower panels exclude refraction. 

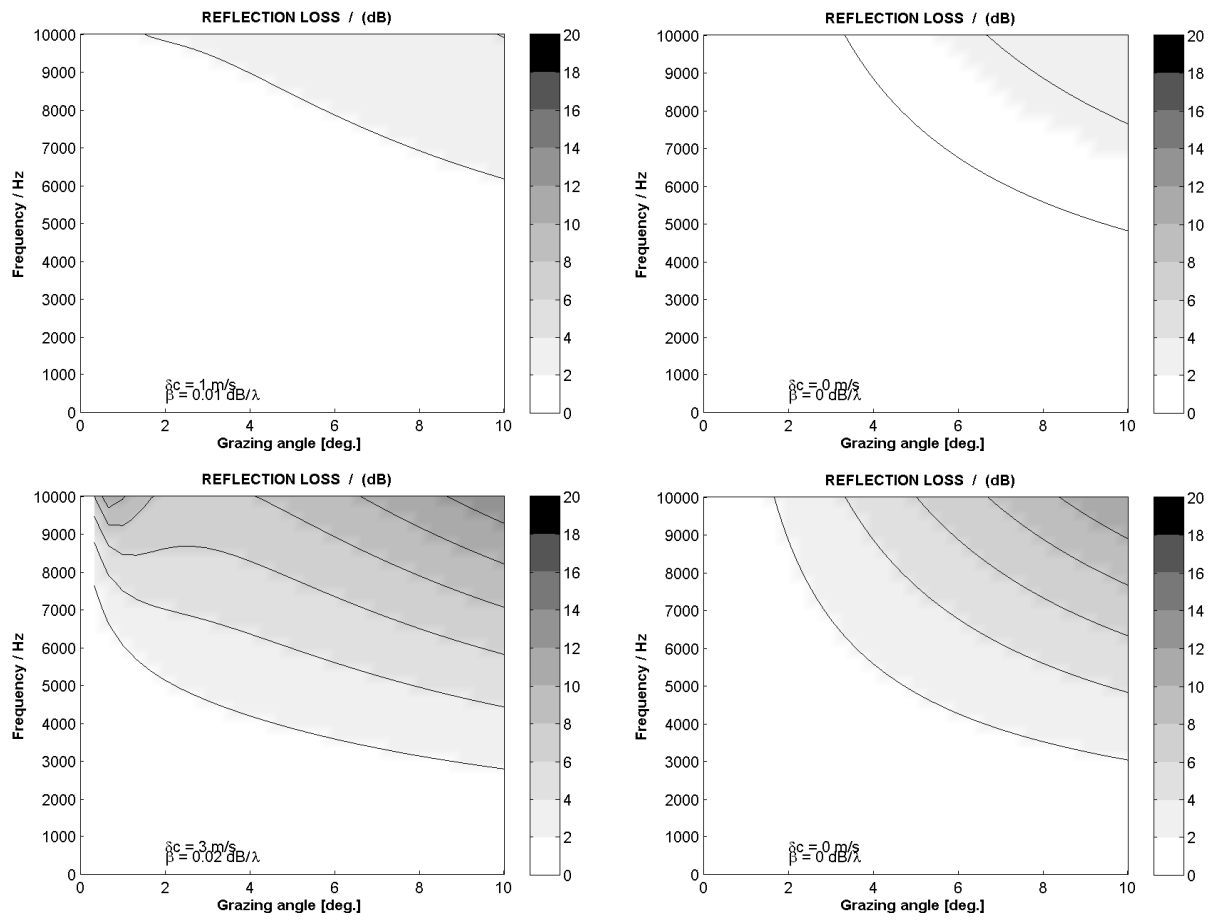

$4 \mathrm{~m} / \mathrm{s}$
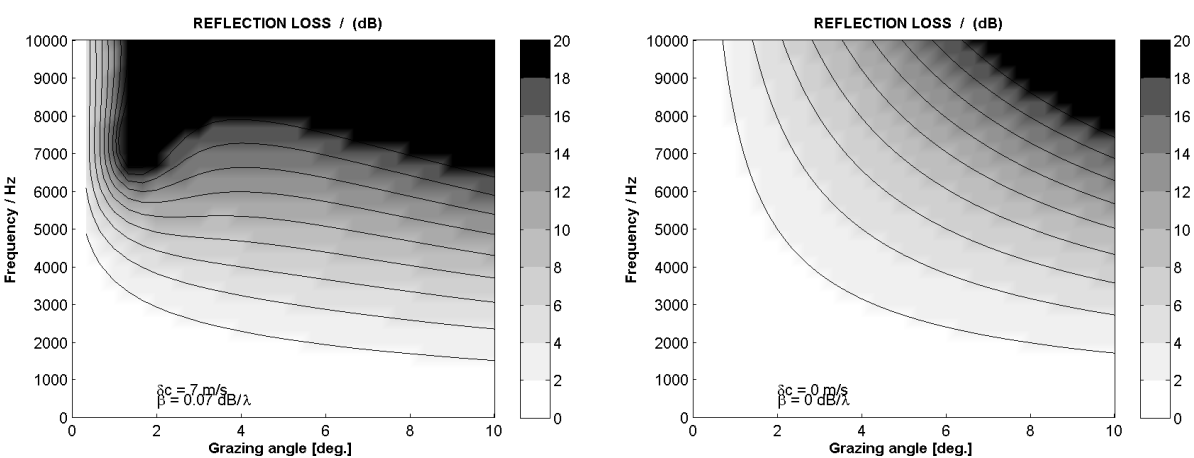

$6 \mathrm{~m} / \mathrm{s}$

$8 \mathrm{~m} / \mathrm{s}$
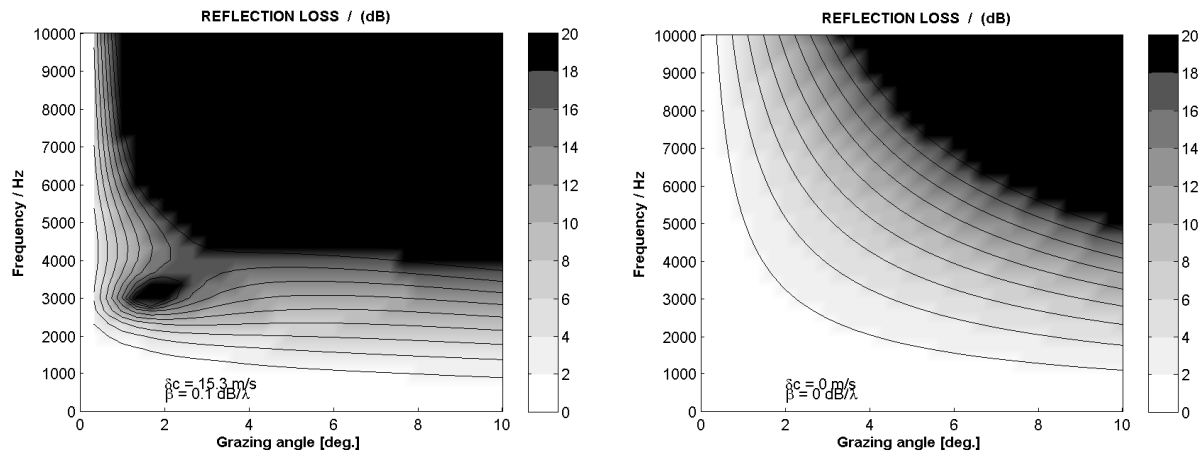

$10 \mathrm{~m} / \mathrm{s}$

Figure 4. Coherent reflection loss $-20 \log _{10}|R|$ (in $\mathrm{dB}$ ) vs. angle and frequency for wind speed between $4 \mathrm{~m} / \mathrm{s}$ (uppermost) and $10 \mathrm{~m} / \mathrm{s}$ (lowermost). Black represents $>18 \mathrm{~dB}$. Left: Effect of rough sea surface with bubbles. Right: Effect of rough sea surface without bubbles. 
Figure 3 shows that the contribution of extinction becomes more pronounced above $4 \mathrm{kHz}$. The visual impression is that refraction is still more important than extinction, although extinction does not seem negligible at frequencies above $4 \mathrm{kHz}$. The applied rough-surface scattering of $0.1 \mathrm{~dB}$ per wavelength is consistent with $10 \mathrm{~m} / \mathrm{s}$ wind speed (cf. Fig. 3 in [13]). In principle, both sound-speed contrast $(\delta c)$ and extinction $(\beta)$ vary with frequency. Figure 3 is intended to give an approximate indication of the relative magnitude of the various physical mechanisms.

The local maxima in the upper panels of Figure 3 are due to destructive interference between the various multipaths. The bubble layer exhibits a sequence of interference nulls close to grazing incidence. This behaviour is similar to that described by Hastrup (1980) [20] for low-speed sediment layers (i.e. sound speed lower than in water).

An important consideration is the wind speed at which effects of bubbles (or the rough surface) start to become important. Consider wind speeds of 4, 6, 8 and $10 \mathrm{~m} / \mathrm{s}$, see Figure 4 . The effect of the bubbles at $8-10 \mathrm{~m} / \mathrm{s}$ is quite large, while the effect at $6 \mathrm{~m} / \mathrm{s}$ and below is less marked.

\section{INTEGRATED MODELLING APPROACH}

Figure 5 shows our integrated approach to the modelling of the sea surface for acoustic channel simulations. Thin-lined boxes represent data and thick-lined boxes represent the used computer codes. The grey box represents an iterative shell script that (time) steps through the consecutive surface-height realizations, e.g. VirTEX [21].

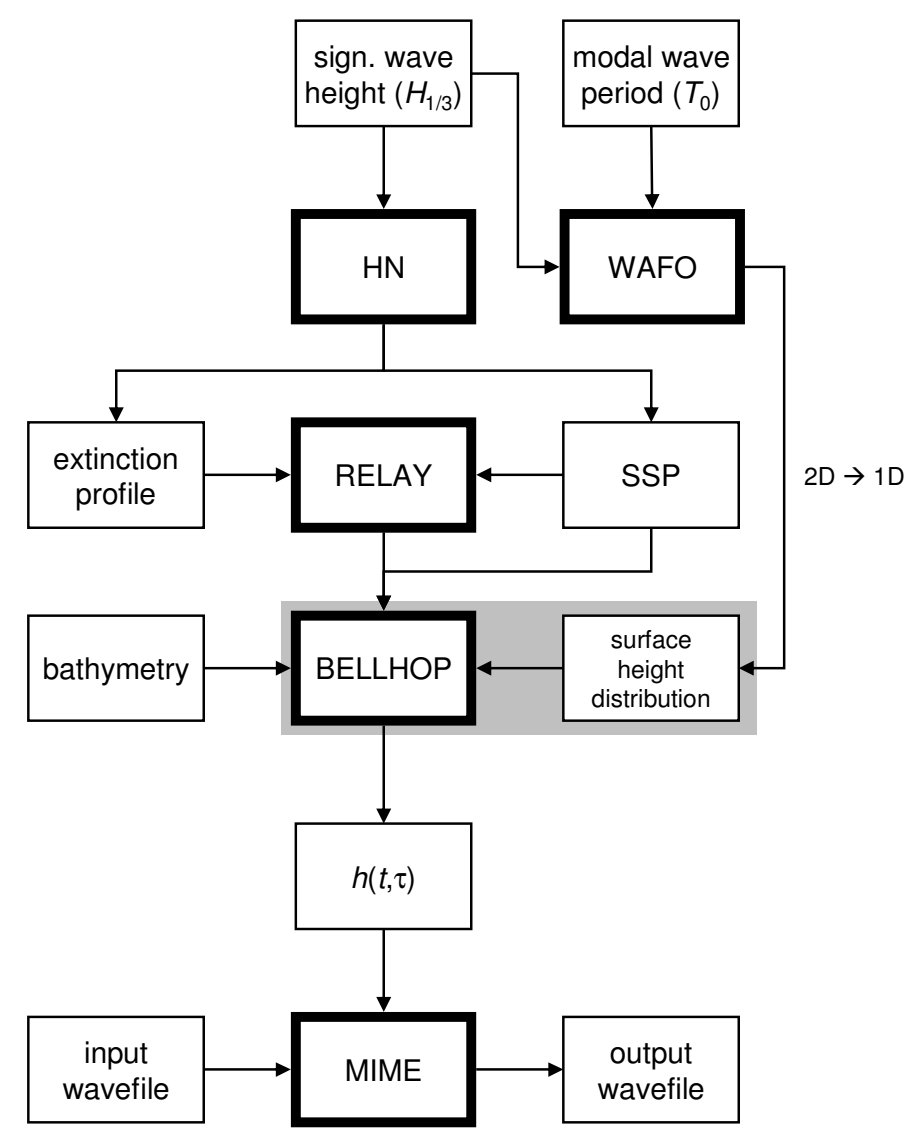

Figure 5. Flowchart showing the dependencies in the integrated sea-surface modelling approach. 


\section{Proceedings of the $11^{\text {th }}$ European Conference on Underwater Acoustics}

The following computer codes are used:

- $\quad$ HN: Hall-Novarini bubble cloud model [14], generating a sound-speed profile (SSP) and a bubble extinction profile for a given wind speed at $10 \mathrm{~m}$ height $\left(v_{10}\right)$, surface roughness $(\sigma)$, or significant wave height $\left(H_{1 / 3}\right)$. (Assumption of a fully developed sea: $H_{1 / 3}=4 \sigma=0.02416 v_{10}{ }^{2}$.)

- RELAY: Computation of (angular) reflection coefficients for the bottom [19] and surface [13]. Uses the SSP and the absorption/extinction profile in the sediment and water column.

- WAFO: Dynamic 2D sea-surface generation for a selected directional wave-height spectrum. Uses also the modal wave period $\left(T_{0}\right)$ when the sea is not fully developed (e.g. for JONSWAP).

- BELLHOP: Computation of impulse response vector $h\left(t_{n}, \tau\right)$ for a given SSP, bottom reflectivity, bathymetry, surface reflectivity and a vertical cross-section (between transmitter and receiver) of the frozen sea surface at time $t=t_{n}$. The ray-tracing computations are performed at $6 \mathrm{kHz}$, which is the centre frequency of the RACUN band (narrowband approximation).

- MIME: A channel simulator that reads the time-stacked impulse response matrix $h(t, \tau)$ and uses it to propagate the communication signal through the channel. In the RACUN project, the Mime channel simulator [22, 2] is used for this purpose.

The BELLHOP ray-tracer will be used in Gaussian-beam configuration [23] in order to produce realistic rough-surface scattering [6] for the detailed surface height distributions produced with the WAFO toolbox.

\section{CONCLUSIONS}

In conclusion, the impact of bubble extinction seems to be significant in the considered frequency band of $4-8 \mathrm{kHz}$ and comparable to refraction. Consequently, if the wind speed is high enough to create bubble clouds (i.e. $6 \mathrm{~m} / \mathrm{s}$ or greater), then extinction due to the bubbles needs to be accounted for in addition to the refraction. This implies that both the sound speed and the bubble extinction profile of the air-water mixture should be incorporated in the propagation model. The bubble extinction is accounted for through the surface reflection coefficients. Modelling of forward scattering, of which the importance increases with wind speed and frequency, is not yet included, and we are interested to learn about the validity of this approximation for the RACUN conditions (frequency band $4-8 \mathrm{kHz}$, wind speed up to $10 \mathrm{~m} / \mathrm{s}$ ). Furthermore, in order to assess the error made by the applied narrowband approximation, a comparison with an alternative propagation model (e.g. parabolic equations) is planned for some well-defined benchmark testcases [24].

\section{ACKNOWLEDGEMENTS}

We kindly acknowledge the fruitful discussions with M. Zampolli on the piece-wise frozen approach to the sea surface modelling.

This investigation has been performed in the framework of the European Defence Agency (EDA) project RACUN (Robust Acoustic Communication in Underwater Networks). The RACUN project is part of the EDA UMS programme (European Unmanned Maritime Systems for MCM and other naval applications), and is funded by the Ministries of Defence of the five participating nations: Germany, Italy, Netherlands, Norway and Sweden

\section{REFERENCES}

1. J. Kalwa, The RACUN project: Robust Acoustic Communications in Underwater Networks An Overview, Oceans 2011, Santander (2011)

2. P.A. van Walree, Trond Jenserud and M. Smedsrud, A discrete-time channel simulator driven by measured scattering functions, IEEE J. Sel. Areas Comm. 26, 1628-1637 (2008) 


\section{Proceedings of the $11^{\text {th }}$ European Conference on Underwater Acoustics}

3. M.A. Ainslie, Principles of Sonar Performance Modelling, Springer Praxis Books (2010)

4. S.A. Thorpe, On the clouds of bubbles formed by breaking wind-waves in deep water, and their role in air - Sea gas transfer, Phil. Trans. R. Soc. Lond. A 304, 155-210 (1982)

5. M.B. Porter, The BELLHOP manual and user's guide, preliminary draft (31 January 2011), http://oalib.hlsresearch.com/Rays/HLS-2010-1.pdf

6. M. Siderius and M.B. Porter, Modeling broadband ocean acoustic transmissions with timevarying sea surface, J. Acoust. Soc. Am. 124, 137-150 (2008)

7. P.A. van Walree, Channel sounding for acoustic communications: techniques and shallowwater examples, FFI-rapport 2011/00007 (2011)

8. W.J. Pierson and L. Moskowitz, A proposed spectral form for fully developed wind seas based on the similarity theory of S.A. Kitaigorodskii, J. Geophys. Res. 69, 5181-5190 (1964)

9. K. Hasselmann, T.P. Barnett, E. Bouws, H. Carlson, D.E. Cartwright, K. Enke, J.A. Ewing, H. Gienapp, D.E. Hasselmann, P. Kruseman, A. Meerburg, P. Müller, D.J. Olbers, K. Richter, W. Sell and $\mathrm{H}$. Walden, Measurements of windwave growth and swell decay during the Joint North Sea Wave Project (JONSWAP), Dtsch. Hydrogr. Z. A8(Suppl.), No. 12 (1973)

10. D.E. Hasselmann, M. Dunckel and J.A. Ewing, Directional wave spectra observed during JONSWAP 1973, J. Phys. Oceanogr. Vol. 10, 1264-1280 (1980)

11. WAFO - a Matlab toolbox for analysis of random waves and loads, Tutorial for WAFO v2.5, Lund University, Sweden (2011), http://www.maths.Ith.se/matstat/wafo

12. P.A. Brodtkorb, P. Johannesson, G. Lindgren, I. Rychlik, J. Rydén and E. Sjö, WAFO a Matlab toolbox for analysis of random waves and loads, Proc. 10th Int. Offshore and Polar Eng. Conf. (2000)

13. M.A. Ainslie, Effect of wind-generated bubbles on fixed range acoustic attenuation in shallow water at 1-4 kHz, J. Acoust. Soc. Am. 118, 3513-3523 (2005)

14. R. van Vossen and M.A. Ainslie, The effect of wind-generated bubbles on sea-surface backscattering at $940 \mathrm{~Hz}$, J. Acoust. Soc. Am. 130, 3413-3420 (2011)

15. M.V. Hall, A comprehensive model of wind-generated bubbles in the ocean and predictions of the effects on sound propagation at frequencies up to $40 \mathrm{kHz}$, J. Acoust. Soc. Am. 86, 11031117 (1989)

16. R.S. Keiffer, J.C. Novarini and G.V. Norton, The impact of the background bubble layer on reverberation-derived scattering strengths in the low to moderate frequency range, J. Acoust. Soc. Am. 97, 227-234 (1995)

17. J.C. Novarini, R.S. Keiffer and G.V. Norton, A model for variations in the range and depth dependence of the sound speed and attenuation induced by bubble clouds under wind-driven sea surfaces, IEEE J. Oceanic Eng. 23, Vol. 4 (1998)

18. G.V. Norton and J.C. Novarini, On the relative role of sea-surface roughness and bubble plumes in shallow-water propagation in the low-kilohertz region, J. Acoust. Soc. Am. 110, 29462955 (2001)

19. M.A. Ainslie, Reflection and transmission coefficients for a layered fluid sediment overlying a uniform solid substrate, J. Acoust. Soc. Am. 99, 893-902 (1996)

20. O.F. Hastrup, Some bottom-reflection loss anomalies near grazing and their effect on propagation in shallow water, in W.A. Kuperman and F.B. Jensen (Eds.) Bottom-Interacting Ocean Acoustics (pp. 135-152), Plenum Press, New York (1980)

21. J.C. Peterson and M.B. Porter, Virtual Timeseries Experiment (VirTEX) - Quick Start, Distributed with VirTEX (8 July 2011)

22. R. Otnes, P.A. van Walree and T. Jenserud, Validation methods for underwater acoustic communication channel simulators, Proc. UAM 2011, Kos, Greece (2011)

23. M.B. Porter and H.P. Bucker, Gaussian beam tracing for computing ocean acoustic fields, J. Acoust. Soc. Am. 82, 1349-1359 (1987)

24. M. Zampolli, M.A. Ainslie and P. Schippers, Scenarios for benchmarking range-dependent active sonar performance models, Proc. Institute of Acoustics (edited by M.A. Ainslie), Vol. 32, Part 2, pp. 53-63 (2010) 